\title{
A Proposal on Gas Leakage Detection System using ARM and Zigbee Technology
}

\author{
Vipin Balyan \\ Cape Peninsula University of Technology, Cape Town, South Africa \\ balyanv@cput.ac.za \\ Received: $3^{\text {rd }}$ December 2020, Accepted: $5^{\text {th }}$ February 2021, Published: $26^{\text {th }}$ February 2021

\begin{abstract}
Smart homes are becoming more commonplace around the world in the last few years. Affordability has made it possible for many people and organisations to automate their daily tasks, such as turning on the lights and setting the thermostat. With this project, we hope to develop a gas leak detector utilising an LPG gas sensor and connect it to Zigbee technology for quick data transmission. This will let us deliver protection in real time for our community.
\end{abstract}

Keywords: ARM, Automation. Gas, Leakage, Zigbee

\section{Introduction}

The weather has the most significant impact on your health issues in daily life. Environmental and industrial concerns related to pollution are handled honestly to raise public and employee health awareness of the environmental danger. Carbon monoxide (CO), cooling oil gas (CO2), and liquefied petroleum gas (LPG) are all colourless and odourless because of incomplete combustion. As a result, gas detector equipment is needed to provide on-demand updates on the safety status. Carbon monoxide (CO), sometimes known as the "silent killer," is a poisonous gas that, if inhaled by a person for an extended period of time, can cause permanent brain damage or even death. Toxicological effects of carbon monoxide (CO) are influenced by the concentration of the gas and the length of exposure. Low CO concentrations, like high CO concentrations, can be lethal for short periods of time if exposed for an extended length of time. Fires are the most prevalent source of CO. Even in modest amounts, it can cause dizziness and headaches within a few hours (e.g. 100 ppm). Extremely short-lived headaches and vertigo (for example, $3200 \mathrm{ppm}$ ), followed by 30 minute deaths. An unconscious state is followed by death within three minutes after a few breaths in extremely high concentrations (e.g., $12800 \mathrm{ppm}$ ). The gas or fireplace is a common cause of indoor $\mathrm{CO}, \mathrm{CO}_{2}$ and $\mathrm{CH}_{4}$ leaks. $\mathrm{CO}$ is made up of a combination of coal gas and natural gas. Combustion of industrial or cosmetic materials releases $\mathrm{CO}, \mathrm{CO} 2, \mathrm{CO} 2$ and alkanes. The vapours themselves are hazardous, and the concentration of these gases can quickly ignite, posing a danger to a number of objects. The location of the gas tank or pipeline is usually fixed, making it easier to inspect, but the location of the fireplace is unpredictable and hence harder to inspect.

Propane and butane are combined to create fluid petroleum gas (LPG), which is extremely flammable. Ethaneoil has a distinctive smell, making it easy to spot leaks. It's odourless air, so you won't notice it. LPG is now one of the substitute fuels. When the time is right. In light of rising diesel and petrol prices, LPG has become increasingly popular as a vehicle fuel source. In 1910, Dr. Walter Snelling became the first person to create LPG. It is a dry and dried mixture of hydrocarbons. LPG can be used in a variety of ways, including for cooking, heating, lighting, and transportation. It can be utilised for a variety of purposes as a result. LPG demand is increasing at a rapid rate. Since LPG has a high calorific value and produces less smoke, it is frequently utilised in automobiles. Natural gas is another commonly utilised residential fuel. The primary issue here is gas leakage into the atmosphere. When breathed, the gases can cause asphyxia since they are heavier than air. Explosions are caused by gas leaks in the atmosphere. Clean energy is produced when natural gas and LPG are burned, although leakage is a significant issue. The death toll from LPG explosions has risen sharply in recent years. When it comes to gas leaks, those with a poor sense of smell could not notice a low concentration of the gas. This necessitates the use of a high-security system. Gas leakage is prevented by the current arrangement. A tragic example of gas leaking occurred in India and Japan with the tragedies of Bhopal and 
Chernobyl. There has never been a gas leak disaster as bad as this one. Detecting and preventing gas leaks is critical, but so is preventing leaks in the first place.

Hence we have developed the system to be able to detect and prevent LPG leaks. By using sensors, gas leak detection is able to spot potentially dangerous gas leaks. We plan to integrate a low-cost gas sensor, MQ6, in a microcontroller-based system to detect several flammable gases. The sensitivity and response time of this sensor are excellent. ARM Microcontroller used to notify the user when gas concentrations exceed the permissible limits.

\section{Literature Survey}

LPG is a non-smoking fuel widely utilised in household, industrial and agronomic applications for cooking, heating systems and other heating needs. LPG implies liquefied petroleum gas consisting of propane or butane or both of propane and butane. mixtures are formed of LPG. A study indicated that smoking 400 cigarettes a day is similar to traditionally cooking with firewood or cow dung. The major goal of cooking the meal, including blowing air in the firewood 'chulha' and smoke breathing, is to create a lot of effort and patience. The LPG cookery as fuel reduces the detrimental effect of use of fireboard and cow dung and the usage "LPG saves indoor waste." This traditional cooking approach was overcome. Government of India launches a mechanism for free connection to rural and urban BPL families on 1May 2016.

According to the latest study, the yearly growth of LPG usage climbed from 9 percent to 9.8 percent from 201516 to 2016-17, and the growth of LPG consumers increased from 10.2 percent to 16.2 percent during the same period. With the development of LPG connection and consumption, it is vital to educate the users with the safety measure to avoid any mishap related to LPG as it is extremely flammable, poisonous and has a danger of fire or explosion. In addition to this, it is a need for a system which can detect and also prevent from leakage of LPG. From a survey throughout the period from "2010" to "2106" it may be established that 17 percent of fire accidents are attributable to LPG leaking. There is a necessity of safety systems that gives safety assurance and conducts safety actions in the absence of users. Commonly it is neglected as an imperative need for safety but since there are risks from this, there should be a safety system implemented for worst case circumstances.

The major purpose of this study is to identify LPG during leakage and takes safety actions in the absence of the consumers. There are two main goals for this project: first, to identify the presence of LPG at a harmful level and to alert the staff of a gas leakage via an alarm and LED indication. Suffocation or explosion of LPG cylinder from ignition can be avoided by utilising solenoid valves that automatically cut off gas flow via the gas pipe line/cylinder. Miniaturizing and manipulating gas leak detection techniques have been used extensively to counteract the harmful impacts of gas leaks.

Several studies have examined the occurrences of gas leak-related incidents and published statistical data. An energy-aware gas sensing system utilising wireless sensor networks was described in 2012 by Somov et al. and it consisted of four components: an energy-aware gas sensor node, a relay node with wireless actuator, and a network coordinator. The core of the WSN is the network coordinator. The IEEE 802.15.4 standard and the ZigBee specifications are used to support the network's wireless communication. Additionally, the network coordinator is responsible for informing a network operator or an emergency service via the Ethernet network or by using a GSM/GPRS modem to transmit a message. Network coordinators are able to deactivate the source of gas emission through a wireless actuator when they receive a warning message from a sensor node.

Design and Development of a Flexible Reliable Smart Gas Detection System" was devised by Bhattacharjee et al. in 2011. With its base station, wireless sensor array, and intelligent wireless alarm unit, this system provides unmatched dependability, adaptability, and uninterrupted sensing. Sensor handover and wireless threshold fixation are only a few of the advanced protocols that are used to attain these results. One pyro-electric infrared sensor (PIR) boosts the sensor node's sensing intelligence with three gas sensors, one temperature sensor, and one pyro-electric sensor Peripheral interface controller (PIC) 16f877A based centrally embedded platform is used to digitise and analyse the detected data, and a pair of 433 and $315 \mathrm{MHz}$ ASK wireless modules are used to communicate wirelessly. It is possible to create a highly secure gas detection system by the encoding and decoding of sensed data.

The most significant failures in environmental protection have occurred as a result of technological advancement and changes in how we live. This has led to an increased focus on environmental contamination control and monitoring systems. With relation to human health, environmental and industrial challenges have 
prompted a sense of responsibility and responsiveness. Accidents involving huge amounts of liquefied petroleum gas (LPG) are common. Because of this, detecting and notifying of gas leaks is of paramount importance. The modern answer requires innovative technologies that are precise and have the most competent quantity system. Detection and alert systems for gas leaks in homes, enterprises, autos, and other places are critical. Only the wireless sensor network system [6] can be used to create a gas leak detection system that is as sensitive as this, and it is a novel class of computing, a new location in information technology, and it is composed of a large number of low-cost micro sensor nodes deployed in a monitored region. Nodes integrated into the network can inspect, collect, process, and send data on perceptible items inside the network's coverage area by determining and controlling parameters. In sensor networks, data transmission, signal processing, and hardware operation all use significant amounts of energy to some degree.

Because of the spread nature of wireless sensor networks, they become more intrusive in the industrial, agricultural, and military industries. To avoid the dangers of gas leaks, this cutting-edge technology was developed. Internet of Things (IoT)-enabled gas monitoring, booking and leak detection Kumar Keshamoni and Sabbani Hemanth are the authors. This journal discusses the most prevalent difficulty that we face in our daily lives, which is the emptying of our GAS containers. We're bringing this paper to raise awareness about how much lighter gas can be, and to place an IOT-based gas order.

\section{Research Methodology}

Existing Method: One-dimensional first-order hyperbolic partial differential equations drive the flow dynamics in a leak detection system that uses an adaptive observer. It is presumed that just the pipe's intake and outflow have measurement capabilities. The leak size estimation problem can be solved independently of how the leak is spread along the pipe, according to convergence properties. This is critical to developing a simple leak estimating technique. To demonstrate the system's ability to detect, quantify, and locate leaks, simulations are presented.

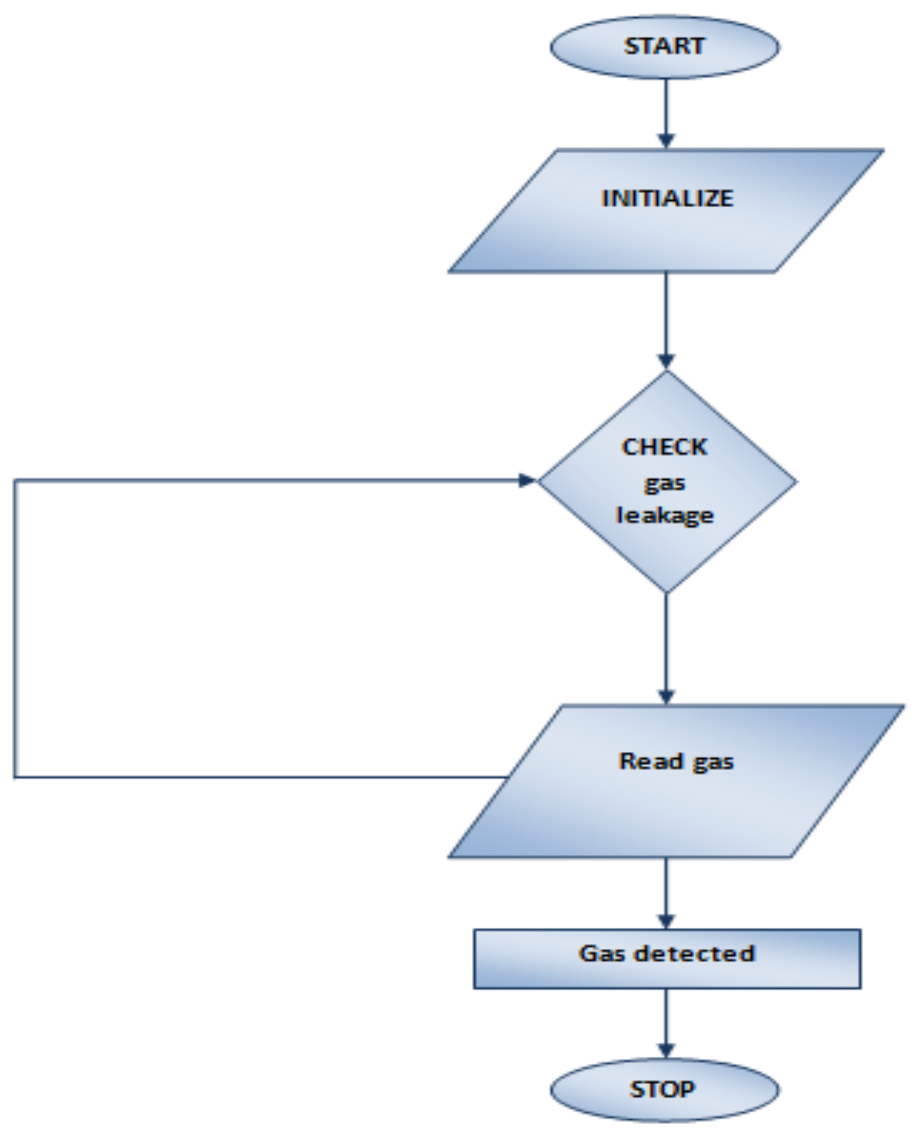

Fig 1: Flow Chart of Existing method 
Data not received accurately.

$>$ No protocol architecture.

$>$ No communication device.

No monitoring system.

\section{Proposed Method}

$>$ Avoiding industrial safety systems failure by keeping an eye out for leaks of liquefied petroleum gas (LPG). This system uses a gas sensor to detect and monitor LPG leaks, and it uses a buzzer and zigbee to deliver an alert message to the user when a leak is detected. LPG concentration in the air is constantly monitored by this system, and when it reaches a predetermined threshold, an alarm is triggered and data is supplied from the monitoring area. Wireless communication is used to notify the user of a gas leak via the monitoring component of the proposed system. It regulates the system simultaneously so that you don't have to think about it. Data receiving is very high

$>$ Wireless communication

$>$ Automatic control \& Alert system

In pipelines, major mishaps are caused by the transportation of fluids. Leaks from pipelines in the oil and gas industry can cause both environmental and economic harm. These are some of the driving forces behind the creation of an effective leak detection system, together with the demands of environmental authorities. The primary goal of this initiative is to prevent fires and harmful circumstances in the environment by monitoring industrial fluid leaks. Using low-power ARM microcontrollers and ZIGBEE methods, as well as sensors that sense the leakage driver unit, these issues can be handled.

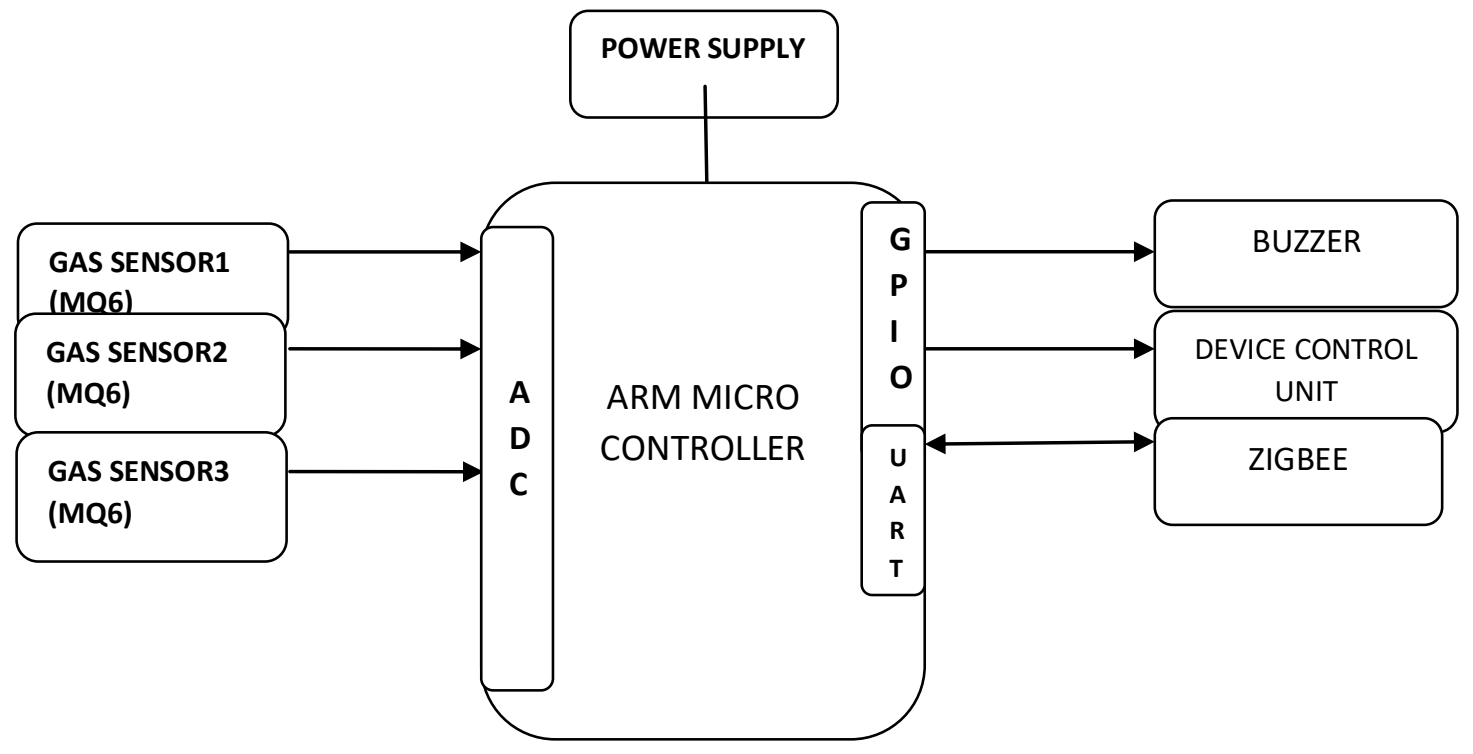

Fig 2: Block diagram of Proposed Method

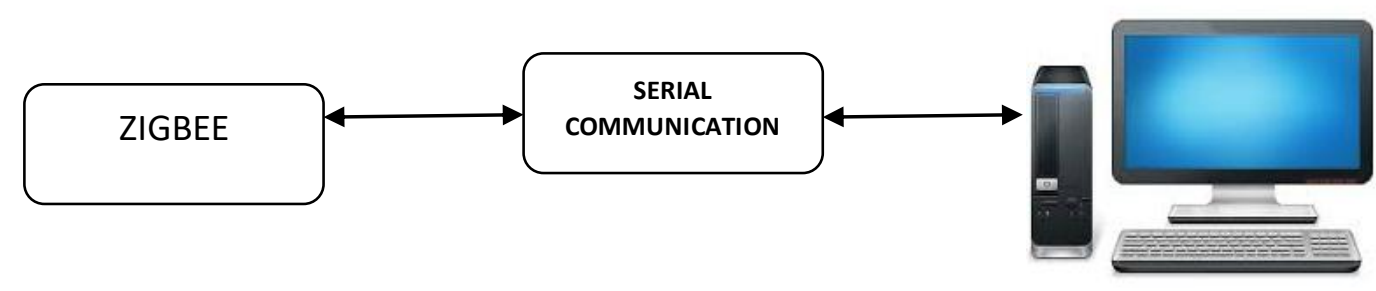

Fig 3: Monitoring section 
Wireless Sensor Networks (WSNs) are a hot topic in computer network research and have the potential to be a significant money-maker in the future. The processing power, storage, bandwidth, and energy available to sensor nodes are all constrained. Security in sensor networks is difficult because of this constraint. Embedded computing and wireless communication are becoming increasingly popular in applications like wireless gas sensing systems because of the proliferation of small, low-cost, and miniatured embedded processors, radios, sensors, and actuators that are often combined on a single chip.

Hardware and software should be created together because sensor nodes and co-ordinators demonstrate embedded philosophy. The microcontroller must interact with the real environment in order to accomplish this predetermined task. Embedded software is necessary for this purpose. Due to the use of an Arduino microcontroller in the current WSN sensor nodes, all software necessary to run the system is written in Python using the Arduino IDE, ZAP 2.0.5. Embedded sensor nodes should be able to operate independently. An appropriate operating system is required for this. The current system uses a super loop to meet the requirements of an operating system. Assemblers and compilers are only one of the development toolbox required for firmware creation. For the current system, which uses XBee for wireless communication, the firmware should include the ability to communicate wirelessly.

\section{Conclusion}

Sensors in the air detect LPG gas leaks, and if they exceed the limit of safety, the ARM microcontroller activates a buzzer and send an alert to the monitoring section through ZigBee. In the event of a hazardous or abnormal situation, this user will be alerted to take the appropriate action. We can avoid gas leak-related accidents with the help of this equipment. We will use GPRS and GSM on the microcontroller to locate the leakage precisely in this project. In addition to informing adjacent stations in the industry, the warning message is broadcast across the airwaves. The leakage can be easily located in the field because to the latitude and longitude measurements. Since the recent explosion of compact and low-powered hardware platforms coupled with sensing, computing, and wireless communications, wireless sensor network design and development has become increasingly popular and useful across many industries (WSN).

\section{References}

1. C. Verde, "Minimal order nonlinear observer for leak detection," Journal of Dyn. Systems, Measurement and Control, vol. 126, pp. 467-472, 2004

2. C. Verde, "Multi-leak detection and isolation in fluid pipelines," Control Engineering Practice, vol. 9, pp. 673-682, 2001

3. E. Hauge, O.M. Aamo and J.-M. Godhavn, "Model-based monitoring and leak detection in oil and gas pipelines," SPE Projects, Facilities \& Construction, vol. 4, no. 3, pp. 53-60, 2009

4. E. Hauge, O.M. Aamo and J.-M. Godhavn, "Model based pipeline monitoring with leak detection," Proc. of the 7th IFAC Symposium on Nonlinear Control Systems, Pretoria, South Africa, August 22-24, 2007

5. J. Salvesen, Leak Detection by Estimation in an Oil Pipeline, MSc Thesis, NTNU, 2005

6. K. Hodne, Leak detection in two-phase oil and gas pipelines by parameter- and state estimation, MSc Thesis, NTNU, 2008

7. L. Billmann and R. Isermann, 'Leak detection methods for pipelines," Automatica, vol. 23, no. 3, pp. 381385,1987

8. M. Hou and P. M"uller, "Fault detection and isolation observers," Inter- national Journal of Control, vol. 60, no. 5, pp. 827-846, 1994 
9. M. Krstic and A. Smyshlyaev, "Backstepping boundary control for first- order hyperbolic PDEs and application to systems with actuator and sensor delay," Systems \& Control Letters, vol. 57, pp. 750-758, 2008

10. N. Bedjaoui and E. Weyer, "Algorithms for leak detection, estimation, isolation and localization in open water channels," Control Engineering Practice, vol. 19, pp. 564-573, 2011

11. O.M. Aamo, "Disturbance rejection in $2 \times 2$ linear hyperbolic systems," IEEE Trans. on Automatic Control, vol. 58, no. 5, pp. 1095-1106, 2013

12. O.M. Aamo, J. Salvesen, and B.A. Foss, ”Observer design using boundary injections for pipeline monitoring and leak detection," Proc. of the 2006 International Symposium on Advanced Control of Chemical Processes, Gramado, Brazil, April 2-5, 2006

13. P.-S. Murvay, I. Silea, "A survey on gas leak detection and localization techniques," Journal of Loss Prevention in the Process Industries, vol. 25, pp. 966-973, 2012

14. R. Vazquez, M. Krstic and J.-M. Coron, "Backstepping boundary stabi lization and state estimation of a $2 \times 2$ linear hyperbolic system," Proc. of the 50th IEEE Conference on Decision and Control and European Control Conference, Orlando, FL, USA, 2011, pp. 4937-4942 